\title{
Preliminary Discussion on Contemporary Screen Design
}

\author{
Chengyuan Ren \\ School of Art and Fashion \\ Tianjin Polytechnic University \\ Tianjin, China 300387
}

\author{
Yuting Mei \\ School of Art and Fashion \\ Tianjin Polytechnic University \\ Tianjin, China 300387
}

\author{
Huiqing Guo \\ School of Art and Fashion \\ Tianjin Polytechnic University \\ Tianjin, China 300387
}

\begin{abstract}
The screen has a centuries-old history. It has both decoration function and practicability. It is not only an indispensable part of Chinese ancient interior design, but also a unique form of furniture in modern people's living space. This paper is divided into three parts to discuss the screen. The first part mainly summarizes the historical development and historical function of the screen. The second part introduces the decorative effect and application of the screen in modern space environment. The third part summarizes the new breakthroughs in all fields of the modern screen design and my design practice of sand painting screen.
\end{abstract}

Keywords-screen; decorative; practicability; modern environment space; sand painting screen

\section{INTRODUCTION}

Literally, "screen is considered as a barrier". Screen is often placed in the interior to keep out the wind, separate from space, and keep out the sight. Relative to other furniture, screen has no complicated structure, but it becomes the essential article in daily life. Giving consideration to beauty and practice, it occupies an important position in the development history of traditional Chinese furniture. Screen has the long history. It was presented in a royal palace at the earliest and it was the symbol of power in ancient times. As a unique furniture form in modern society, screen plays a crucial role on building the indoor environment atmosphere by virtue of special practicality and cultural connotations.

\section{OVERVIEW OF SCREEN}

\section{A. Historical Development Situation of Screen}

The origin of screen should be traced back to the Western Zhou Dynasty or earlier. At that time, screen was called as "Di" or "Yi". According to the recording in the Rites of Zhou, "a mat was established in the royal residence for the emperor." "Di" was the board behind the chair and it was decorated by lines [1][2].

In the Warring States Time, screen has already had the high craftsmanship and there was ornamental small screen
[3]. Painted screen earthed from the Chu Grave in Xinyang (a city in Henan) had several snack-like dragons (Panli) twining on the seat. With smooth and natural workmanship, these snack-like dragons were lifelike, as shown in "Fig. 1".

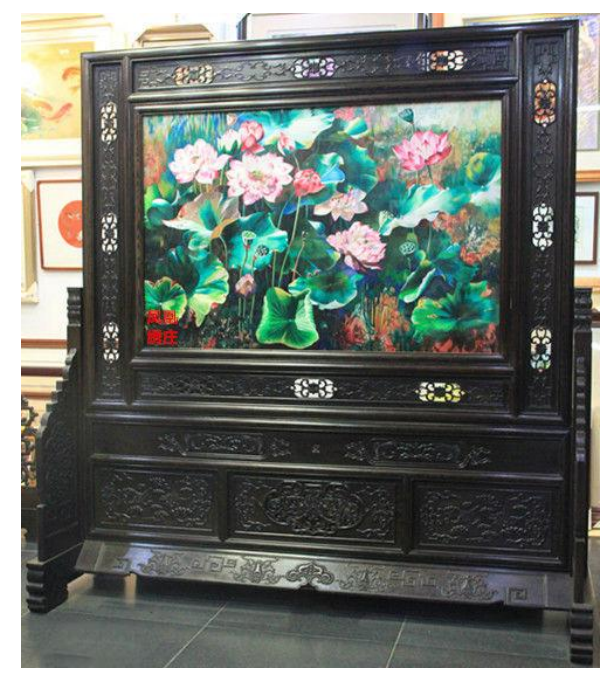

Fig. 1. Screen style in the Warring States Time.

In the Han Dynasty, screen was even generally used. Wealthy and influential families were equipped with screen. Good or bad screen also embodied the status of users. Screen types and categories were also slightly changed by comparing with the previous dynasty. The independently single form of screen was changed into the curved screen with multi-fan combination and it was also called as joint screen,as shown in "Fig. 2". The furniture could be considered as the perfect combination of folding screen and bed and it gave both considerations to keep out the wind and lean on or against function. 


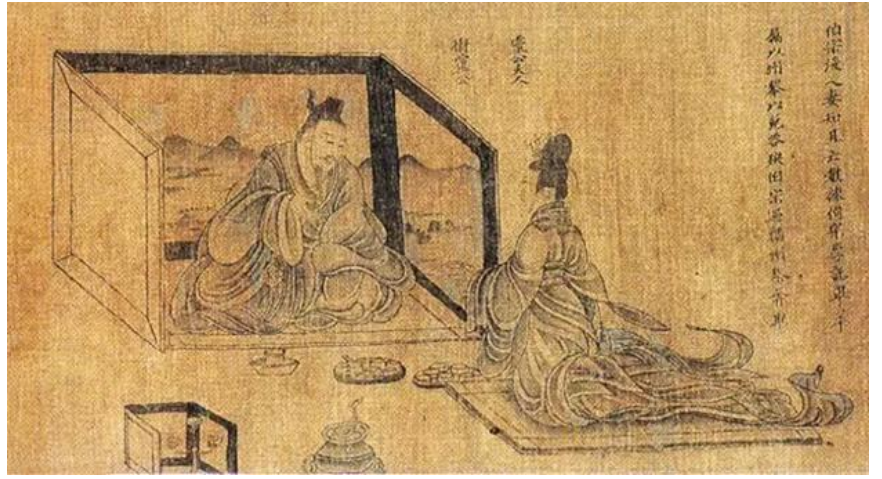

Fig. 2. Screen in the Han Dynasty.

During the period from Wei and Jin Dynasties to Sui Tang and the Five Dynasties, multi-fan screen describing landscapes started to be popularized and it had no need to install pedestal, but just needed to open a fan to stand upright. In addition to display, the screen could keep out the wind, as shown in "Fig. 3". During the period of Sui Tang and the Five Dynasties, screen had various categories. Refined scholars wrote articles and painted on the screen and such a style was prevailing. Screen not only could be used as the decorative element in the interior, but also could separate from the space. During the period, there were different types of screen, which were main force for furnishings, as shown in "Fig. 4". The role of separating from the space for the screen was also stood out during the period of Han and Tang Dynasties [4].

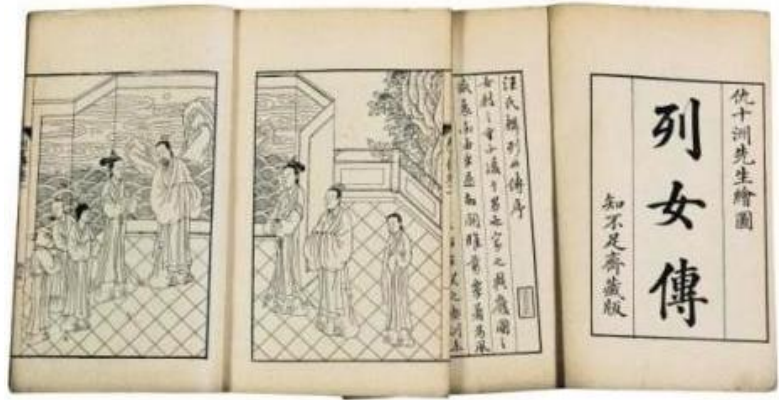

Fig. 3. Typical screen style in the Wei and Jin Dynasties.

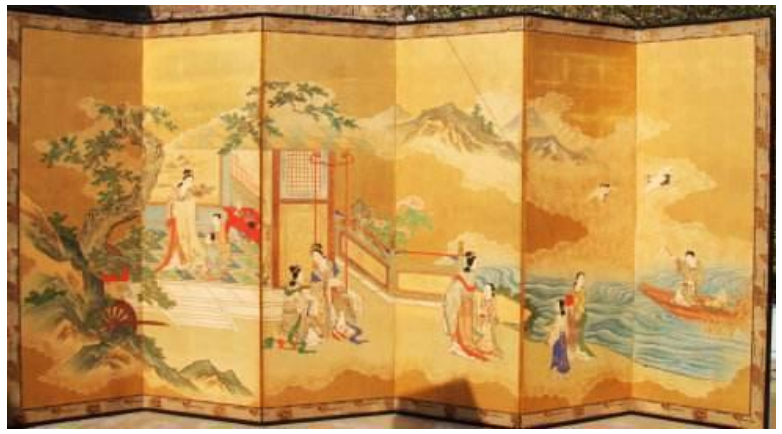

Fig. 4. Typical screen style in the Tang Dynasty.

Song Dynasty paid attention to rational thinking, advocated to worship the nature in the process of creation and pursued for order and law. With the lifestyle change in
Song Dynasty, screen was increased with the height of seat, thus the pedestal of screen was developed into bridge-type pedestal with strip ledger. At the moment, screen had the simple and plain modeling structure and there were few tedious decorations, as shown in "Fig. 5". With the elegant style, it made people feel the beauty of freshness and nature [5].

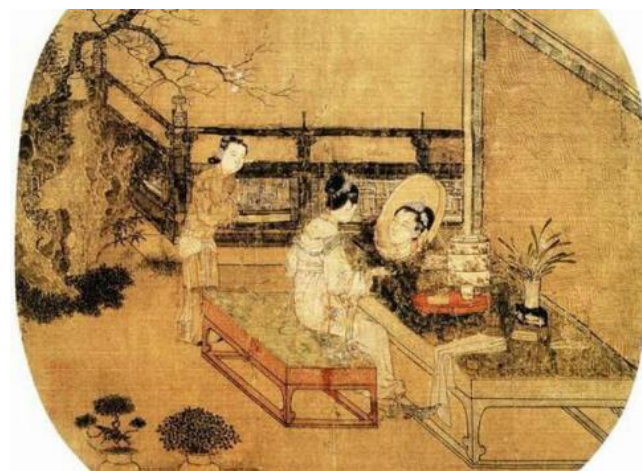

Fig. 5. Typical screen style in the Song Dynasty.

In the Ming Dynasty, screen was developed into the stable and mature period. The screen could be divided into unfolded screen with pedestal, table screen and curved screen. There was mainly the unfolded screen with pedestal, showing the exquisite and meticulous figures. Screen fabrication was equipped with advanced hardwood, especially for dalbergia odorifera and padauk. The wood grain was natural and fresh. By comparing with the previous dynasty, screen technology in Qing Dynasty was tedious. It probably could be divided into unfolded screen with pedestal, hanging screen, folding screen and table screen. Yongzheng and Qianlong periods were of great prosperity, which could be shown in numerous examples. The sumptuous degree was even superior to the previous dynasty. The most representative one was the screen behind the emperor's throne in the imperial palace, as shown in "Fig. $6 "$.

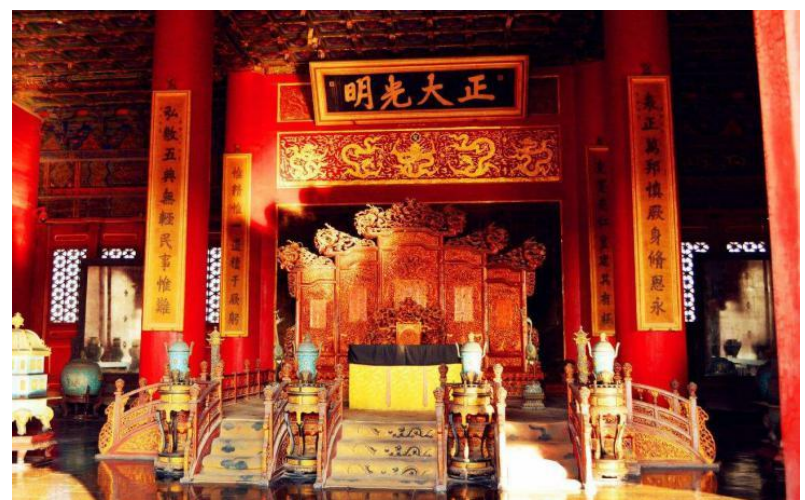

Fig. 6. Typical screen style in the Ming Dynasty

In modern times, Chinese society had the earth-shaking changes, flowing western culture into China. People's thought and lifestyle were greatly impacted by western culture. The phenomenon of "western learning introduced into China" was increasingly obvious. The furniture industry gradually became western-style. At the moment, no matter 
for the modeling, functions or the decorative technology, screen was greatly improved. The artistic carving was obviously increased in screen design. The style of screen was also developed into multiple styles from the single Chinese style, such as Baroco style, Rococo style and Victoria style, which were embodied in the screen production at that time. With the changes of styles, screen was more than used by royal households and nobility. Gradually, it could be used by common people.

\section{B. The Role and Function of the Screen}

1) To Keep out the Wind and Shield the Sight: To keep out the wind is the original function of the screen. Screen was applied by imperial palace of the Western Zhou Dynasty at the earliest. The Emperor of Zhou faced to the south and suffered from cold current in the back, thus screen was used to withstand cold wind at that time. It could meet basic life demands for people.

In addition to keep out the wind, screen also could shield the sight. In ancient times, people placed the screen in the axis of buildings to compensate for leakage in the open space. Afterwards, people often placed the screen in the entrance of interior space with the constant development of economic society, especially for the large space, such as banquets and exhibition rooms, etc., to keep out the wind and shield the sight, as shown in "Fig. 7".

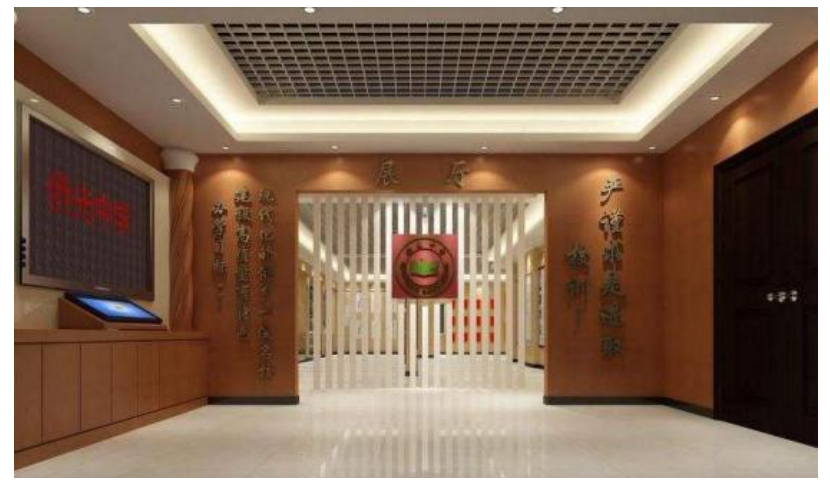

Fig. 7. The interior screen at the entrance.

2) To Separate from the Space: Screen is the mediator between interior space structure and indoor furnishings. Screen localizes the former and provides he suitable space position for the later furnishings. The application screen can make the closed and static space become mutually communicated and exchanged, so as to integrate gradually. In other words, interior can be really combined with the nature to live up to "connection with separation". This has been the leading function of the screen through the ages.

Mobility of the screen brings about flexibility to divide interior space. For example, in the last years of Qing Dynasty, in order to master the national power, Empress Dowager Cixi ordered someone to hang a curtain and sat behind the emperor to hold power in secret. This was called as "holding court from behind a screen". In modern society, there are possessed with removable screens in public space, such as hospitals and stations, dividing space to enhance concealing and provide convenience for daily life.
3) To Embody Cultural Connotations and Build Empty Beauty in the Space: Chinese ancient traditional ideology was greatly affected by Confucianism, striving for coordination between human and nature, theory that man is an integral part of nature and interaction between heaven and mankind. There is no doubt that screen production technology in ancient China was also integrated with the "theory that main is integral part of nature." Screen drawings were inspired from the nature. With the themes of flowers, birds, fishes and insects, mountains and rivers, it showed that ancestors respected for nature and dreamed of nature. In Chinese ancient traditional culture, men and women had different status. When there were customers at home, women were not allowed to meet in person, thus, screen played the virtual role of shield to build the romantic feeling of "covering the face by embracing pipa".

The gardening art in ancient China has acquired the considerable achievements and the application of screen could play a crucial role. The hallow screen windows found out a view skillfully. With the coordination between wood texture and natural green landscapes, indoor and outdoor scenes could echo."

\section{DEDUCTION OF THE SCREEN IN MODERN INTERIOR DESIGN}

\section{A. Decorative Effect of Screen}

With the development of modern society and science, there are increasing numbers of types and categories in screen. The exquisite screen design needs the combination of various elements and technologies. The current screen design is decorated through the texture changes and colors of materials, so that it can display all kinds of styles, so as to meet demands of environment space.

\section{B. Application of the Screen in Environment Space}

In ancient times, there were single screen categories and texture, tedious decoration and complicated craftsmanship. The utilization also had the strict hierarchical system. In modern society, social status tends to be equal, thus screen utilization becomes "popularization". Undoubtedly, the technology and application range also become increasingly diversified.

1) Application in Dining Space: In modern society, a restaurant is more than the dining space. What's more, it is a good choice for party and negotiation. As a result, the dining space not only provides dining behaviors for people, but also provides a place for expression and communication between humans, this kind of screen as shown in "Fig. 8".

Screen has the functionality and decoration. Reasonable application of the screen in the dining space can regulate the space size and regulate the empty space. This is good for building the catering space atmosphere and playing a crucial role on setting off the thematic style [6]. 


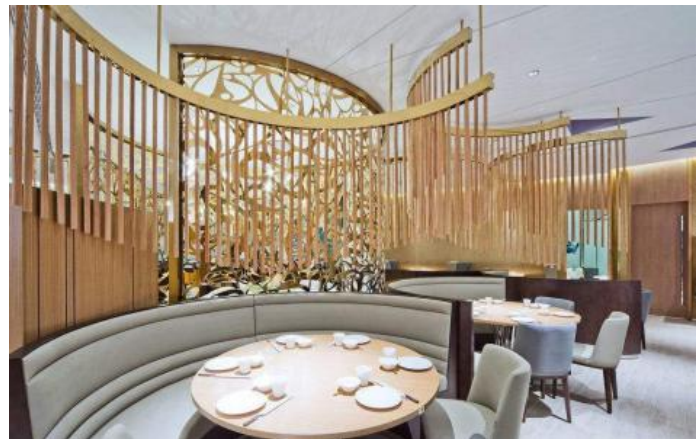

Fig. 8. The screen in the dinning space.

2) Application in Living Space: The screen separation is also widely utilized in modern living space, as shown in "Fig. 9". In the living space, a living room often integrates with receiving visitors, dining and entertainment. In order to plan the space without damaging the integrity, designers often conduct "open-type treatment" for screen separation, so that it reach the better space effects.[7]

To place the screen in a large bedroom is also a good choice. According to Fengshui Theory, "master bedroom shouldn't be too large, because geomancy is easy to weakened and scattered." The rest space in the master bedroom is separated from make-up and office space, as shown in "Fig. 10". This not only can ensure privacy of rest space, but also will be good for sleep and health.

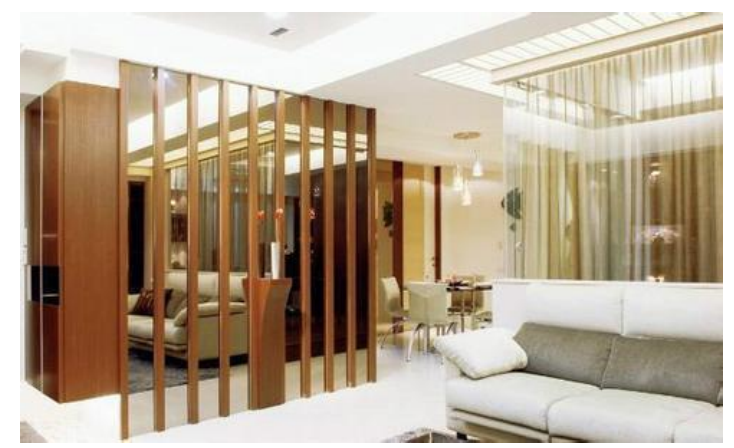

Fig. 9. The screen in the living space.

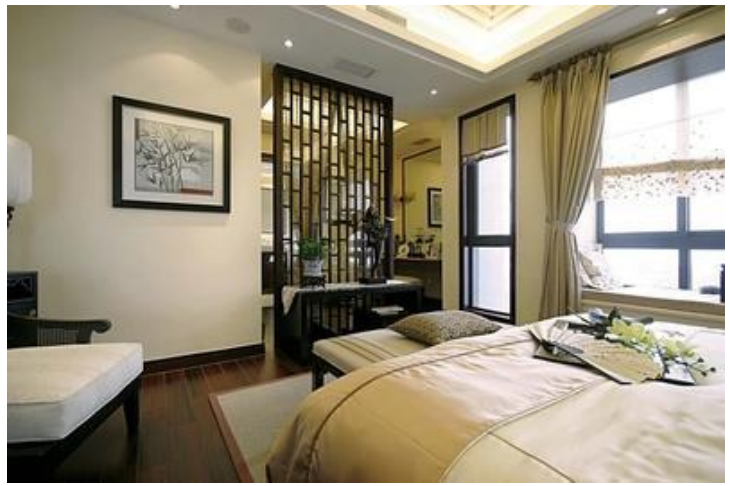

Fig. 10. The screen in the bed room.

3) Application in Office Space: An office is a primary area for daily work and study. Office design affects human mood directly. It is directly connected with work efficiency, thus separation design in an office not only should meet mental demands, but also should consider individual privacy and separate from suitable space to meet visitors [8].

In the office space, creative separation is used to separate from space. The iron stand in industrial style is matched with potted plants in different modeling to refresh and regulate work atmosphere, so that human mood can be relaxed and relieve working pressure, his kind of screen as shown in "Fig. $11 "$.

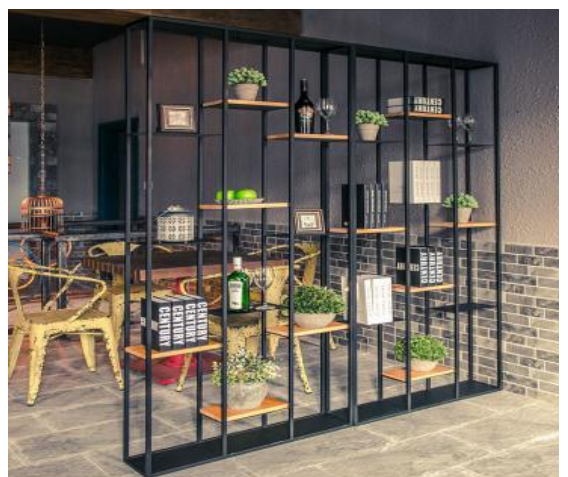

Fig. 11. The screen in the office space.

4) Application in Commercial Space: The commercial space mainly includes commodity space, entertainment space and display space. According to different space demands, separation is also slightly different in design. For the commercial space giving priority to female shopping, such as clothing shops, accessory stores and cosmetic stores, the separation design is provided with the soft lines and colors for women. For the commercial space favored by men, such as car exhibition clubs and science parks, separation should embody epochal character and functionality of products. The screen for this function is shown in "Fig. 12".

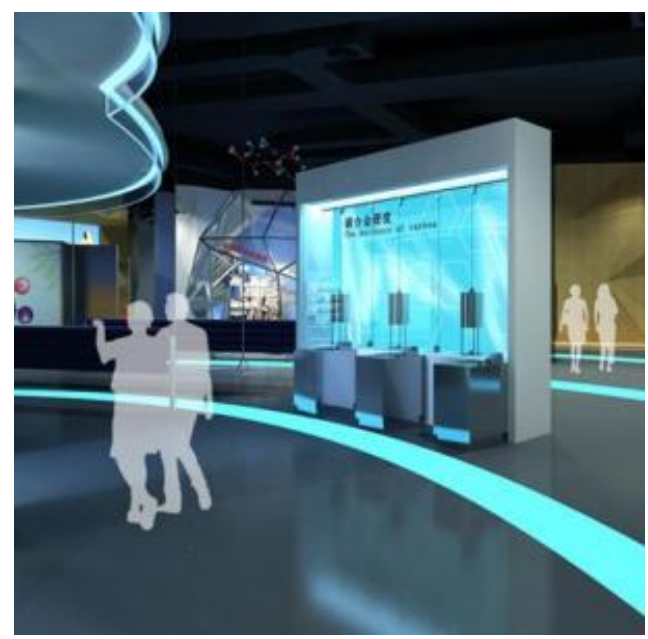

Fig. 12. The screen in the office space. 


\section{NEW EXPLORATION OF SCREEN DEVELOPMENT}

\section{A. Diversified Style Performance}

In the past, antique-and-curio shelves were often applied in Chinese-style interior design, with wood texture and single brown colors. With the constant development of interior design, the style of antique-and-curio shelves also becomes increasingly diversified, as shown in the "Fig. 13". Based on the antique-and-curio shelves with sufficient sense of design, black is interspersed in the white. It can be placed in the living space or office space to separate space, showing the integration of elegance and calm in modern fashion. In the selection of service space, the design can be applied flexibly to meet sensing demands and mental demands, no matter for high-end and decent minimalism or countryside child-free style favored by women [9].

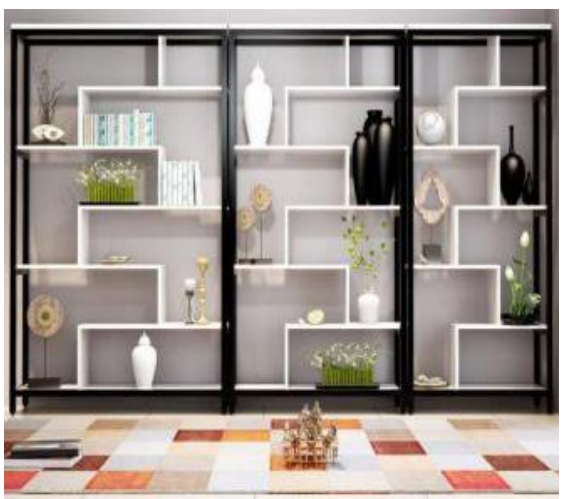

Fig. 13. The application of antique-and-curio shelves.

Breaking through the constraint of traditional screen design for many years, furniture companies in Northern Europe utilize various green plants to separate the interior space, as shown in "Fig. 14". These green plants can be placed in family space or office space, so as to build the relaxed, pleasant and beautiful environment for their daily life and cultivate their taste. In addition, natural plants in a large area are good for purifying interior air, so that people can breathe the fresh air and it is good for human health [10$11]$.

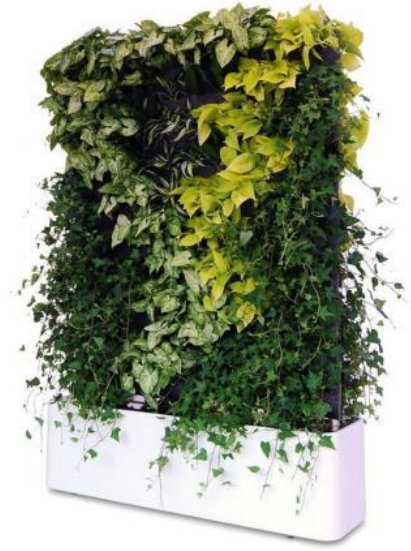

Fig. 14. Application of decorative screen in plants.

\section{B. Multifunctional Application}

Application of fish tank is a kind of fashion in modern home decoration, as shown in "Fig. 15". The space between the living room and the restaurant is separated by the fish tank. Visually, water is used as the separation to increase the house area, decorate the space and make residents pleasant. In addition, moisture evaporated from the fish tank can increase moisture in the living room to relieve the drying environment. It is good for fitness of residents.

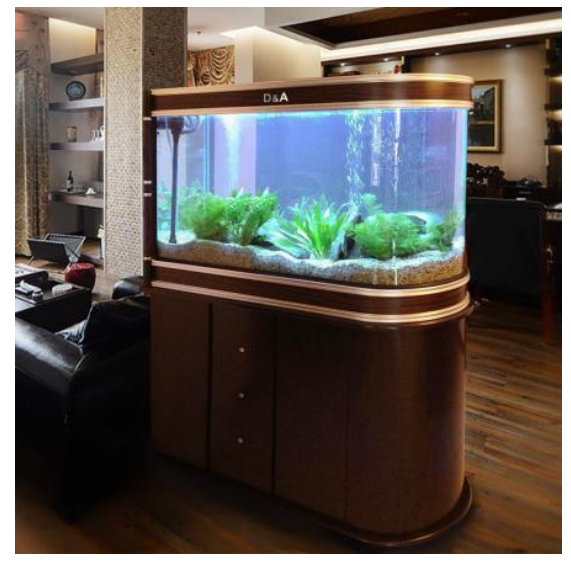

Fig. 15. Application of fish tank.

Hallow carved screen is placed in the small-scale room, as shown in "Fig. 16". The screen separates the bedroom from the living room. It is beautiful and magnificent, also plays a decorative role and increase sense of design in the interior space. This not only can ensure privacy of the bedroom, but also can accept coats and hats, providing convenience for human life.

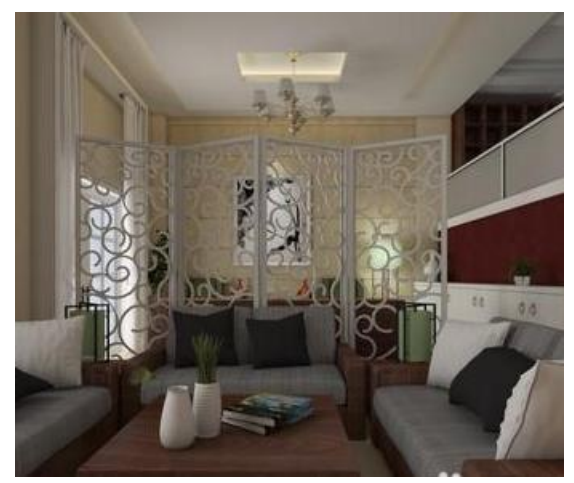

Fig. 16. Hallow carved screen.

\section{Influences of New Materials and New Techniques}

Good screen design is the outcome of materials, science and techniques. The first material of screen design is to select correct materials. There are all kinds of materials for designing modern furniture. Excellent screen design is the organic combination of various materials to meet use demands and spiritual needs in different environment space.

In modern society, screen production materials have various, abundant and extensive categories. Lots of screen design intersects and combines with various materials mutually and organically to make screen full of epochal 
character from the typical ancient style. There is no doubt that every texture has the unique styles and features, such as color, texture, quality, hardness, plasticity, corrosion resistance, and morphology, and so forth. To master features of various textures accurately and apply them in screen design suitably and skillfully is contributed to displaying real beauty of screen design.

Technology is an important link in material creation and development. Without good materials and good technology, it also can't develop the unique charm. As a result, good technology can fully embody unique conception and thinking of creators. The perfect combination and application of new materials and new techniques can embody uniqueness of screen design.

The glass screen separation as shown in "Fig. 17", not only has the good visual effects, but also can increase interior brightness in all directions, thus it seems that small environment isn't separated or divided to some extent, destroying the rigid and occlusive space. The entire space can be vivid and energetic. The good sound insulation effects can ensure that everyone get away from the noisy disturbance and work in the quiet environment. Furthermore, glass also can be combined with other elements to present more design choices.

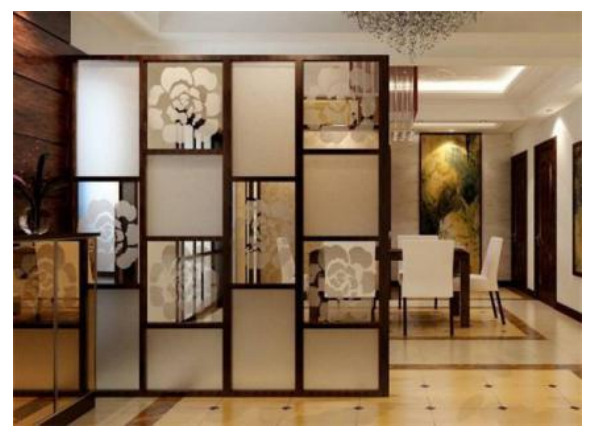

Fig. 17. The glass screen.

\section{Design Practice}

Screen is widely applied in modern society and presented in all kinds of space design. The author explored the screen in multiple aspects from the traditional development to modern development, collected lots of relevant data, combined with the market survey to be enlightened, and attempted to design it boldly, as shown in "Fig. 18".

As a unique artistic form in modern society, common sands can be used to create the beautiful artwork by hands. It conforms to aesthetic appreciation of modern people and has the higher aesthetic value.

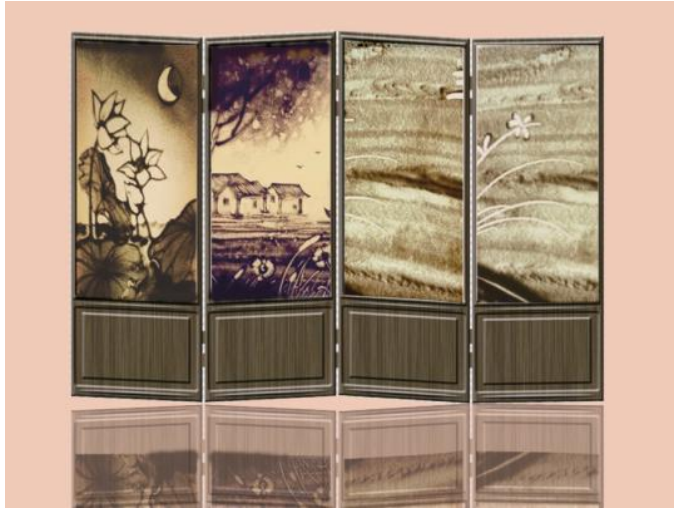

Fig. 18. Sand elements are applied in Chinese-style screen.

Sand elements are applied in Chinese-style screen, which is designed by using new design philosophy, new materials and new techniques. Sand paintings are unrestrained, magnificent and forceful in vision. It is filled with sense of time. Matched with Chinese wood screen, it is the perfect collision of fashion and tradition, thus the entire screen is animato and exuberant. The screen is suitable for home furnishing or can be placed in the public space, such as Chinese restaurants, commercial space and office space, and so on. A large area of "scene" on the screen is enough for people to develop creation in their spare time. Moreover, both of sides can be used for painting. The entire screen design can integrate modern language into traditional elements perfectly, forming the unique style and significance.

In the design of modern screen, the occurrence of new materials and new elements brings about the brand-new creative inspiration to our design, so that screen design can reach the perfect unification of functions and decorative effects, setting off the interior atmosphere of different environment.

\section{CONCLUSION}

Originating from China, screen history is more than thousands of years. It has the profound influences and spiritual connotations and it was the wisdom embodiment of labor workers in ancient times. Gathering the unique Chinese cultural charm, its functions and roles are unique and can't be replaced by other furniture. Screen development changes and affects our daily lifestyle and living habits, thus screen becomes the fashion trend in the decorative industry. In addition, decoration and practical functions of screen are also increasingly important and play a crucial role in separating from the interior space and building artistic space. To sum up, screen is an indispensable part in modern home space and public space.

\section{REFERENCES}

[1] Meiling T, The traditional screen decorative art of the research modern age evolves[D], Hubei University of Technology, 2009.

[2] Yu G, Cate vs harmony, enclosore vs penetration, the application of screen in dinning space[D], Beijing Institute of Fashion Technology, 2010.

[3] Sha Z, Study on screen[D], Nanjing Forestry University, 2008. 
[4] Donghong L, Cultural illumination of chinese ancient screen designing[D], Hunan University Of Technology, 2008.

[5] Ziting Z, The beauty of the screen, study on screen design of Song Dynasty in China[D], Nanjing Normal University, 2014.

[6] Yi F, New function of screen and construction of artistic conception in dining space[D], Shanghai Normal University, 2014.

[7] Hongye L, Space traversing -- study on the application of partition art in modern interior space[D], Shandong Normal University, 2014.

[8] Xue Y, Futing W, Chinese traditional screen and its culture[J], Art Science and Technology, 2014(11):121-121.

[9] Wenyan C, Screen evolution since Han Dynasty[J], Mass Literary Magazine, 2012(14):95-96.

[10] Xia C, The innovation of the screen design research[D], Central South University of Forestry and Technology, 2012.

[11] Hanlun C, Environmental function and application of screen[J], Art and Life, 2012(6):68-71. 